\title{
Preparation of polystyrene-clay nanocomposite by solution intercalation technique
}

\author{
P K PAUL ${ }^{*}$, S A HUSSAIN ${ }^{\dagger}$, D BHATTACHARJEE ${ }^{\dagger}$ and M PAL \\ Department of Physics, Jadavpur University, Kolkata 700 032, India \\ †Department of Physics, Tripura University, Agartala 799 022, India \\ $\ddagger$ CSIR - Central Mechanical Engineering Research Institute, Durgapur 713 209, India
}

MS received 11 November 2011; revised 16 August 2012

\begin{abstract}
Polymer-clay nanocomposites of commercial polystyrene (PS) and clay laponite were prepared via solution intercalation technique. Laponite was modified suitably with the well known cationic surfactant cetyltrimethyl ammonium bromide by ion-exchange reaction to render laponite miscible with hydrophobic PS. X-ray diffraction analysis in combination with scanning electron microscopy gives an idea of structural and morphological information of PS-laponite nanocomposite for different varying organo-laponite contents. Intercalation of PS chain occurs into the interlayer spacings of laponite for low organo-laponite concentration in the PS-O-laponite mixture. However, aggregation and agglomeration occur at higher clay concentration. The molecular bond vibrational profile of laponite as well as PS-laponite nanocomposite have been explored by Fourier transform infrared spectroscopy. Thermogravimetric analysis along with differential scanning calorimetry results reveal the enhancement of both thermal stability and glass transition temperature of PS due to the incorporation of clay platelets.
\end{abstract}

Keywords. Polystyrene; laponite; nanocomposite; Fourier transform infrared spectroscopy (FTIR); basal spacing; intercalation.

\section{Introduction}

In recent times, polymer-clay nanocomposites have attracted much attention for academic and industrial aspects, because they often exhibit remarkable improvement in material properties when compared with pure polymer or conventional micro- and macro-composites (Ray and Okamoto 2003). In this new class of materials, nano-sized inorganic fillers (at least one dimension) are dispersed in polymer matrix to provide improvements such as high tensile strength and modulus (Biswas and Ray 2001), decreased gas permeability and flammability (Bourbigot et al 2000) and increased biodegradability in case of biodegradable polymers (Ray et al 2006). However, optimal performance of such composites is achieved when clay fillers are uniformly dispersed in the polymer matrix. Fabricating such homogeneous mixtures, however, poses considerable synthetic challenge. A major difficulty arises from the inherent structure of the clay particles, which are composed of broad, closely stacked silicate sheets. The diameter of the sheets lies typically between 20 and $200 \mathrm{~nm}$, depending on the specific type of clay and each sheet is roughly $1 \mathrm{~nm}$ in thickness (Wang et al 2006). The spacing (or 'gallery') between the sheets is also in the order of $1 \mathrm{~nm}$, which is smaller than the radius of gyration of typical polymers. Consequently, there is a large entropic barrier that inhibits polymer from penetrating this gap and

\footnotetext{
*Author for correspondence (pkpaul@phys.jdvu.ac.in)
}

intermixing with clay. Even when the sheets are successfully separated and inter-dispersed into the polymer matrix, these high-aspect ratio platelets can form ordered or crystalline structures within the polymers or can phase separate from the matrix material. Hence, to create composites with optimal properties, it is important to consider preparation methods as well as various parameters which reveal how the composition of the mixture affects thermodynamic stability and morphology of the material.

The commonly used layered silicates for the preparation of polymer-clay nanocomposites belong to the same general family of 2:1 layered or phyllosilicates (Ray and Okamoto 2003). Their crystal structure consists of layers made up of two tetrahedrally coordinated silicon atoms fused to an edge-shared octahedral sheet of either aluminum or magnesium hydroxide. Stacking of the layers leads to a regular van der Waals gap between the layers called the interlayer or gallery spacing. This type of layered silicate is characterized by a moderate surface charge known as the cation exchange capacity (CEC), and generally expressed as mequiv/100 g (Alexandre and Dubois 2000). This charge varies from layer to layer and must be considered as an average value over the whole crystal.

Among the various types of nanoclays, laponite is the most abundantly explored natural clay (Hou et al 2004). This layered hydrous magnesium silicate has been widely used in many different fields such as drilling fluids, batteries, cosmetics and paints because of its high degree of structural regularity and low levels of impurities (Eduardo and Pilar 1990; 
Fossum 1990; Velde 1992; Doeff and Reed 1998). Sodium ions between the negatively charged silicate layers are readily exchanged for other ions, which make it possible for polymer molecules to intercalate into the interlayer regions of laponite (Fu and Qutubuddin 2004). Strong interactions between the polymer and the layered silicate in polymerlayered silicate (PLS) nanocomposites lead to the organic and inorganic phases being dispersed at the nanometer level. As a result, nanocomposites exhibit unique properties not shared by their micro counterparts or conventionally filled polymers (Giannelis 1996).

In this present communication, we report the preparation of polystyrene-laponite nanocomposite material using solution intercalation technique. Thermoplastic polystyrene (PS) is a well-characterized hydrophobic polymer. Although there are few reports (Sun et al 2004; Ruggerone et al 2009) on the synthesis of polystyrene-laponite nanocomposites, the present method of preparation is a simple and cost-effective method for preparing such composite materials with improved physical and structural properties. Ravichandran et al (2008) reported the preparation of polymer nanotube composites by similar approach for photovoltaic packaging applications. The main purpose of the present study is to understand the changes in the structural, morphological as well as thermal properties of PS after dispersing the nano-clay of various concentrations. Nano-clayreinforced PS matrix composite prepared by this technique can exhibit improved structural phase and thermal stability. As laponite is only miscible with hydrophilic polymers, it was organically modified by ion-exchange reaction with some alkylammonium ions of cetyltrimethyl ammonium bromide (CTAB) to render this layered silicate miscible with hydrophobic PS. X-ray diffraction (XRD) analysis in combination with scanning electron microscopy (SEM) gives valuable information about the structure and morphology of the resultant nanocomposites. The molecular vibrational profile and thermal properties of synthesized nanocomposites have been interpreted by Fourier transform infrared spectroscopy (FT-IR) and differential scanning calorimetrythermogravimetric analysis (DSC-TGA), respectively.

\section{Experimental}

\subsection{Materials}

The clay mineral laponite was procured from Laponite Inorganics, UK and was used as received. This laponite was actually a synthetic clay (laponite-RD type). The size of the clay platelets was $<0.05 \mu \mathrm{m}$ and its CEC was $0.74 \mathrm{meq} / \mathrm{g}$ determined with $\mathrm{CsCl}$ (Szabó et al 2008). Chemical composition (dry basis) was $\mathrm{SiO}_{2}$ (59.5\%), $\mathrm{MgO}(27.5 \%), \mathrm{Li}_{2} \mathrm{O}$ $(0.8 \%), \mathrm{Na}_{2} \mathrm{O}(2.8 \%)$ and loss on ignition was $8.2 \%$. This type of clay belongs to the group of tetrahedrally substituted 2:1 phyllosilicates (Ray and Okamoto 2003). CTAB $\left(\mathrm{C}_{19} \mathrm{H}_{42} \mathrm{NBr}\right.$, MW = 364.46) was purchased from Aldrich Chemical Company, USA and used without further purification. PS in its solid pellet form was purchased from Polysciences Inc., USA. Triple-distilled deionized water (resistivity, 18.2 $\mathrm{M} \Omega-\mathrm{cm}$ ) and chloroform were used as solvents in this experiment.

\subsection{Preparation of PS-laponite nanocomposites}

Laponite stock aqueous dispersion was first prepared by dispersing a known mass $(1.5 \mathrm{~g})$ into 1 litre of deionized water. This dispersion was sealed and stirred by a magnetic stirrer for at least one week before use. Then aqueous solution of CTAB $(500 \mathrm{mg} / \mathrm{L})$ was mixed slowly with laponite aqueous dispersion and the mixture was again stirred at $50{ }^{\circ} \mathrm{C}$ for $24 \mathrm{~h}$ to attain adsorption maximum. It was then centrifuged at 10,000 rpm followed by repeated washings with deionized water till complete removal of the halide ions. The mixed dispersion was kept aside at room temperature for three days and subsequently, a white precipitate was obtained by filtration. These white precipitates were then

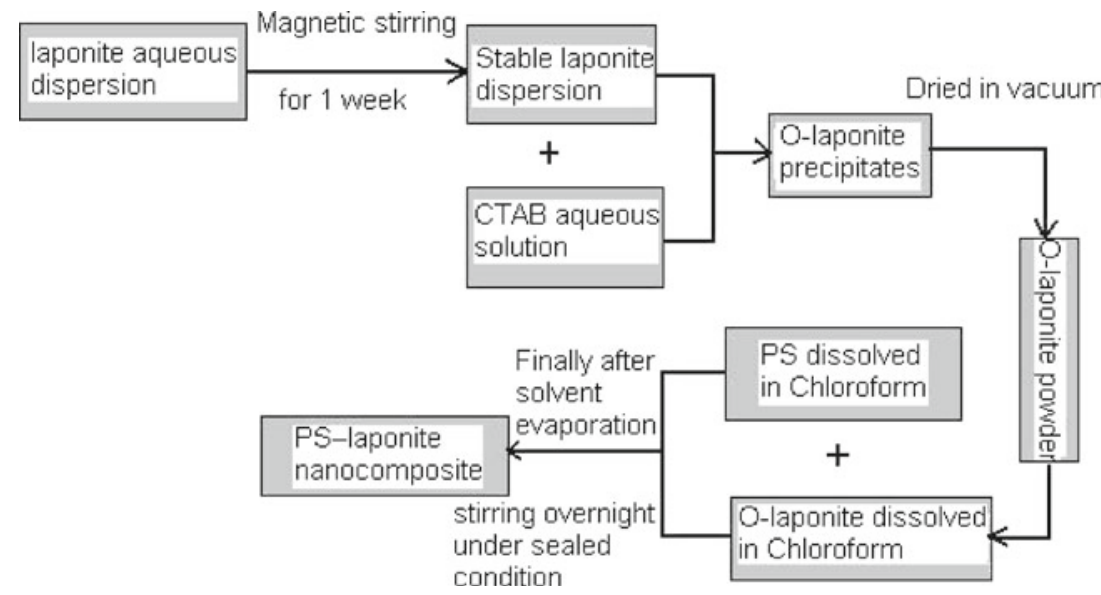

Figure 1. Flowchart of preparation scheme for PS-laponite nanocomposite. 
dried in a vacuum oven at $60^{\circ} \mathrm{C}$ so as to remove the desorbed water molecules and finally made into powder with a mortar and pestle. Laponite clay modified in this way becomes organophilic.

In the next step, these organically modified laponite (Olaponite) particles were dispersed into PS matrix by solution intercalation technique. For this purpose, chloroform solution of PS $(5 \mathrm{mg} / \mathrm{ml})$ was prepared. Different amounts (in wt\%) of O-laponite were taken in borosil beaker and then dispersed in spectroscopic grade chloroform and stirred for $48 \mathrm{~h}$. O-laponite and PS solutions were then mixed and stirred with magnetic stirrer under completely sealed condition. Upon removal of chloroform, composites of PS and Olaponite were obtained. Flowchart for the preparation steps is shown in figure 1.

\subsection{Characterization}

Once the PS-laponite composites were prepared, they were characterized by various analytical tools. To understand the crystalline domains and intercalation of PS between the interlayer spacing of laponite in the composite materials, X-ray powder diffraction data were recorded using an X-ray powder diffractometer (Advanced D8 Diffractometer, Bruker, Germany) with $\mathrm{CuK}_{\alpha}$ radiation (wavelength, $1.54 \mathrm{~nm}$ ) source operated at $40 \mathrm{kV}$ and $120 \mathrm{~mA}$. The scanning speed was $2 \%$ min. Fourier transform infrared spectrophotometer (IR Prestige, Shimadzu, Japan) was used to explore the vibrational signatures of different organic and inorganic species present in the composites. For thermal property measurements, an integrated differential scanning calorimeter (Perkin Elmer DSC-TGA, SDT Q600 V20.5 Build 15) was used. To visualize the phase morphology and intercalated structures in the composites, high resolution field emission scanning electron microscopy (FESEM) (Model S-4800, Hitachi, Japan) was employed.

\section{Results and discussion}

\subsection{Organic modification of laponite with CTAB}

Many of the layered silicates including laponite usually contain hydrated $\mathrm{Na}^{+}, \mathrm{K}^{+}, \mathrm{Li}^{+}, \mathrm{Ca}^{++}$, etc. and so they can be dissolved in water. Obviously, these clays are only miscible with hydrophilic polymers such as polyethylene oxide (Aranda and Ruiz-Hitzky 1992) or polyvinyl alcohol (Greenland 1963). However, for mixing with hydrophobic polymer such as PS, addition of cationic surfactant such as CTAB to laponite through ion exchange reaction makes the clay organophilic. This organic modification actually lowers the surface energy of silicate surface and improves the wetting behaviour of laponite with the polymer matrix into which it will be dispersed. Additionally, long chains of CTAB, with positively charged ends, are tethered to the surface of the negatively charged laponite layers resulting in an increase of clay gallery height (Ray 2006). This also favours the PS to diffuse or intercalate between the layers and eventually separate them resulting in more exfoliated structures (Ray and Okamoto 2003).

\section{$3.2 X$-ray diffraction analysis of formation of PS-laponite nanocomposites}

Intercalation of PS chain into O-laponite layers has been confirmed by the analysis of XRD data of pure laponite, O-laponite (CTAB-modified laponite) and PS/O-laponite composites. Figure 2 shows XRD pattern of pure laponite, CTAB-laponite and PS/CTAB-laponite. These results provide beneficial information about the $d$-spacing of the intercalated hybrids by following Braggs Law, $2 d \sin \theta=n \lambda$, at the observed peak position. In the figure, it is observed that pure laponite shows somewhat broad XRD pattern due to low crystallinity and small particle size (Hanley et al 1997; Blanton et al 1998). It has a broad peak at approximately $2 \theta=5.6^{\circ}$ and is attributed to (001) crystal plane or basal spacing of the clay (Frost et al 2007). Further peaks are present at $2 \theta$ values of $20^{\circ}, 27.5^{\circ}$ and $33.7^{\circ}$ corresponding to (100), (005) and (110) crystal planes, respectively (Tawari et al 2001; Daniel et al 2008). However, when laponite is modified with CTAB by ion-exchange reaction, its characteristic peak, i.e. from (001) plane has been shifted to lower angles corresponding to an increase in $d$-spacing from $15.76 \mathrm{~nm}$ to $24.58 \mathrm{~nm}$. That is, incorporation of CTAB into laponite makes laponite to become more exfoliated structure.

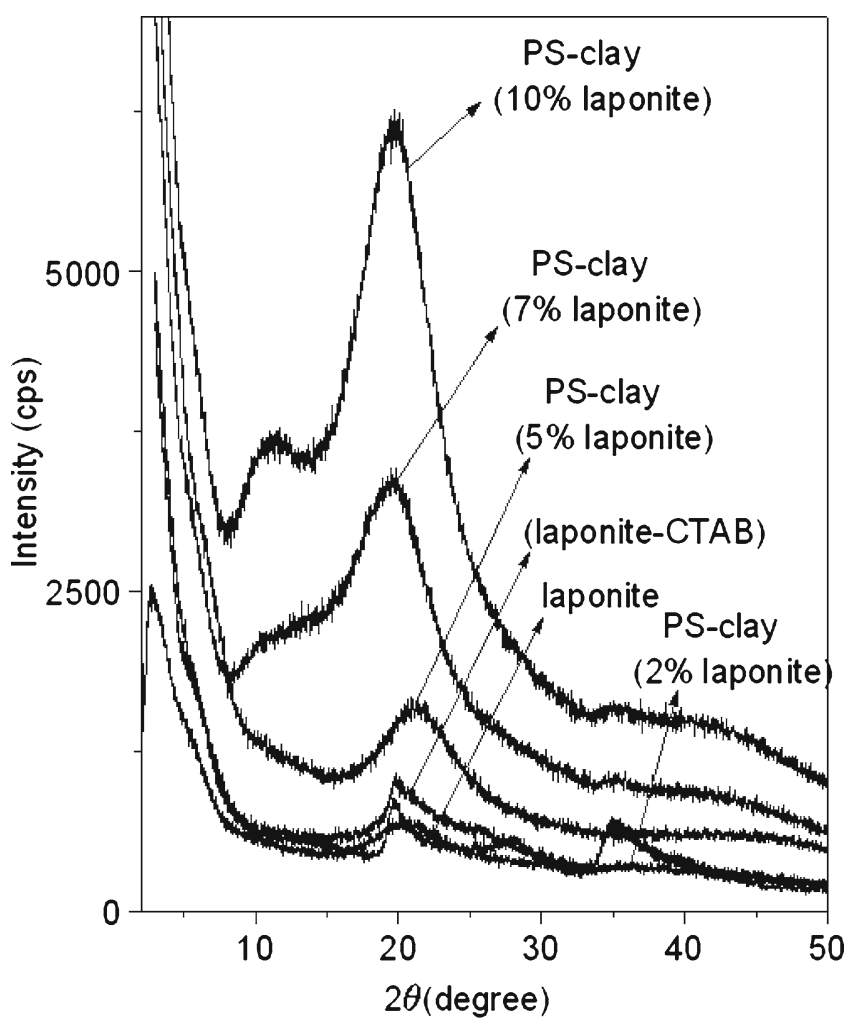

Figure 2. X-ray diffraction pattern of pure laponite, O-laponite and PS/O-laponite composites for different O-laponite contents. 
On the other hand, XRD pattern of PS/O-laponite composites shows somewhat different crystallographic signatures. In case of O-laponite content, $2 \mathrm{wt} \%$ and $5 \mathrm{wt} \%$ in the comoposites, all peaks except $2 \theta=20^{\circ}$ are absent. This certainly indicates that some fractions of the PS chains were intercalated between the interlayer spacing of O-laponite along with some degree of exfoliation of nanoclay platelets present in the nanocomposites. In this way, PS-laponite nanocomposites were obtained. However, with increase in Olaponite content (i.e. for 7 and $10 \mathrm{wt} \%$ ) in PS, aggregation or agglomeration of clay layers increases which is manifested in the occurrence of new peak centred at around $2 \theta=11^{\circ}$. In this situation, individual clay platelets came closer and as a consequence $d$ value decreases. Also the intensity of (100) peaks increases with increase in O-laponite content. As at higher $2 \theta$ values, there are almost no peaks observed, the composites may contain some exfoliated structures.

\subsection{Fourier transform infrared spectroscopy}

Figure 3 shows that FTIR spectra of pure laponite, Olaponite, PS and PS/O-laponite nanocomposites are all mixed with $\mathrm{KBr}$ pellets. FTIR spectrum of pure laponite exhibits a broad structureless band at around 3700$3400 \mathrm{~cm}^{-1}$ in a range of frequencies usually assigned to the stretching and bending of surface hydroxyl groups and

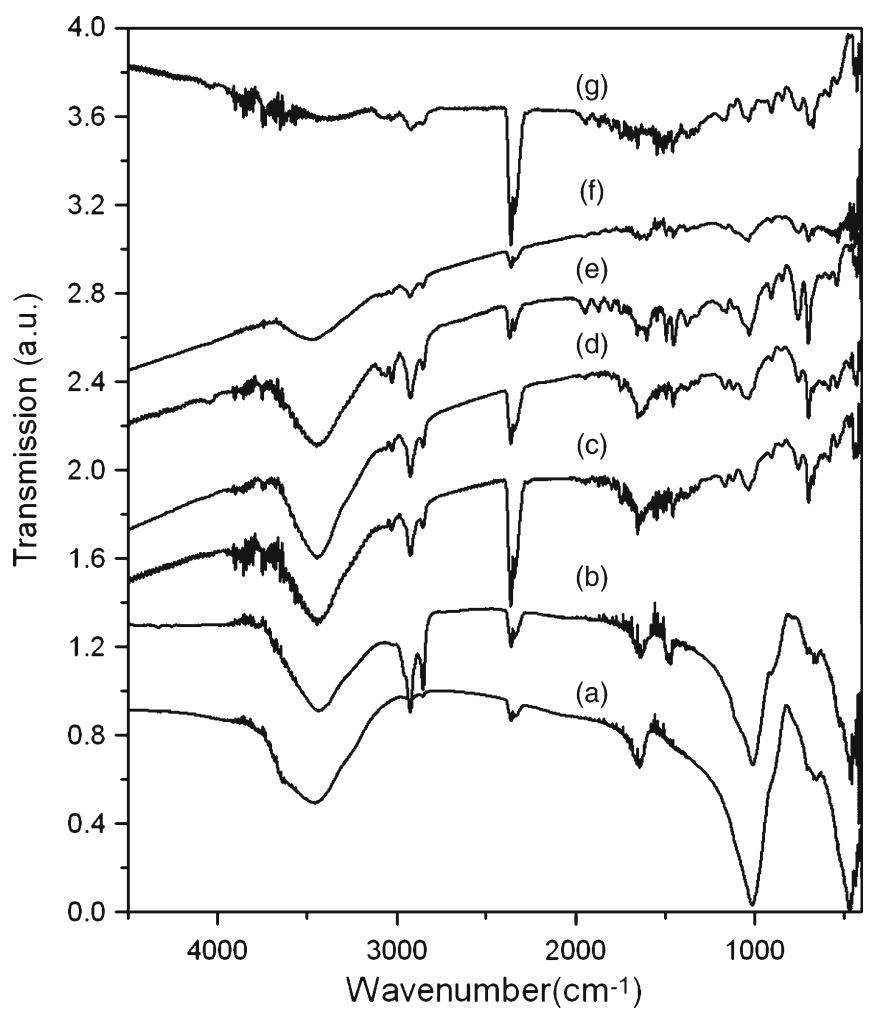

Figure 3. FTIR spectra of (a) pure laponite, (b) CTAB-laponite, (c) PS/O-laponite composites having $2 \mathrm{wt} \%$ O-laponite, (d) PS-Olaponite composites having $5 \mathrm{wt} \%$ O-laponite, (e) PS-O-laponite composites having $7 \mathrm{wt} \%$ O-laponite, (f) PS-O-laponite composites having $10 \mathrm{wt} \%$ O-laponite and (g) pure PS. sorbed water (Guimarães et al 2007). In the higher energy region, peaks are observed at 1001 and $470 \mathrm{~cm}^{-1}$. These are classified as $\mathrm{Si}-\mathrm{O}$ bond and $\mathrm{Si}-\mathrm{O}$ bending vibrations (Fang et al 2009). The peaks observed at $660 \mathrm{~cm}^{-1}$ indicate $\mathrm{Mg}-\mathrm{O}$ bonding (Fang et al 2009). Another peak centred at $1638 \mathrm{~cm}^{-1}$ is ascribed to $\mathrm{Si}-\mathrm{O}$ stretching vibrations of pure laponite.

On the other hand, adsorption of CTAB molecules in the interlayer spacing of laponite is confirmed by the occurrence of peaks at around $2923-2860 \mathrm{~cm}^{-1}$ and at $1475 \mathrm{~cm}^{-1}$. These peaks are due to $\mathrm{C}-\mathrm{H}$ bonds stretching and bending vibrations, respectively in CTAB molecules. Additionally, the transmittance intensity decreases when laponite becomes organophilic after CTAB adsorption.

Figure 3 also shows FTIR spectra of PS/O-laponite composites at various percentages of O-laponite to PS. Interestingly, in all the cases $(2-10 \mathrm{wt} \%)$, the peak for $\mathrm{Si}-\mathrm{O}$ bending vibration $\left(1001 \mathrm{~cm}^{-1}\right)$ was present, but with decrease in intensity. This is due to dispersion of laponite in the composites and also intercalation of PS between the interlayer spacing of laponite. However, all the styrene bands at 700 (C-C out of plane bending vibration), 757, 1022, 1452, 1493, 1601, 2352, 2582, 2924 and $3022 \mathrm{~cm}^{-1}$ are present in the final PS/laponite nanocomposites. Furthermore, intensity of $2352 \mathrm{~cm}^{-1}$ band increases with increasing organo-laponite content.

\subsection{Field emission scanning electron microscopy}

In order to observe the effect of organo-clay addition on the morphology of the prepared composites, scanning electron micrographs (SEMs) of PS-laponite composites having different organo-clay contents along with pure PS are obtained as shown in figure 4. SEM image (figure 4a) of pure commercial PS shows smooth textured lines because of the homogeneity of their structure and there were as such no barriers to stop this smooth propagation. On the other hand, in case of PS-laponite composites (figure $4 \mathrm{~b}, \mathrm{c}$ and d), these smooth textured lines were totally destroyed and random distribution of textures was observed. With the increase in organoclay contents in the composites, the structures become more compact and the porosity of the material increases. It is also evident that inclusion of clay into the polymer matrix brings about some roughness in the resultant structure. For higher organo-clay content (i.e. $10 \mathrm{wt} \%$ ) in PS/O-laponite composites, the structure becomes agglomerated because of the larger extent of aggregation of organo-clay in the polymer matrix. This is also evidenced from XRD analysis of PS/O-laponite as shown in figure 2.

\subsection{Differential scanning calorimetry and thermogravimetric analysis}

Thermal stability of polymeric materials is usually studied by thermogravimetric analysis (TGA). Weight loss due to the formation of volatile products after degradation at high temperature is monitored as a function of temperature as 

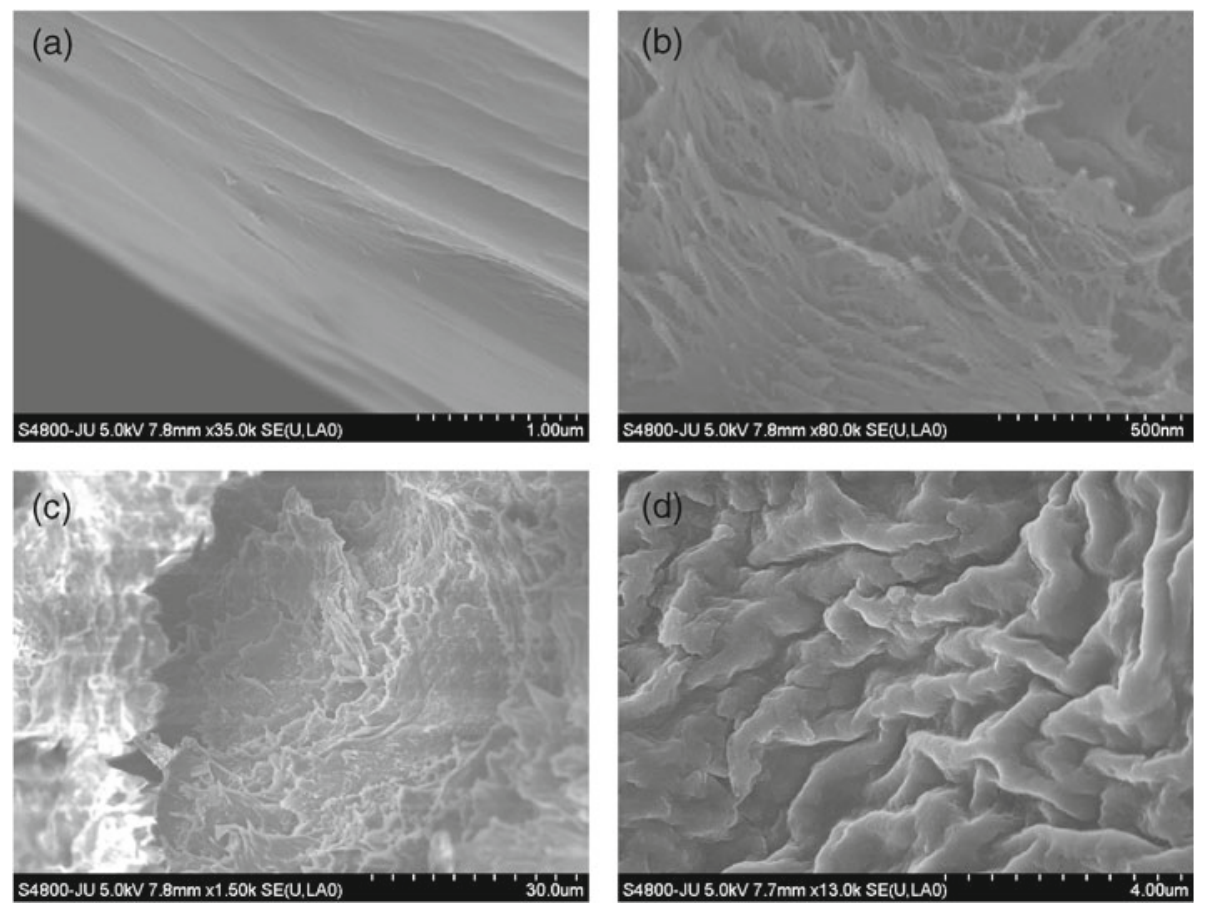

Figure 4. SEM images of (a) pure polystyrene, (b) composite (5 wt $\%$ laponite), (c) composite (7 wt \% laponite) and (d) composite (10 wt\% laponite).

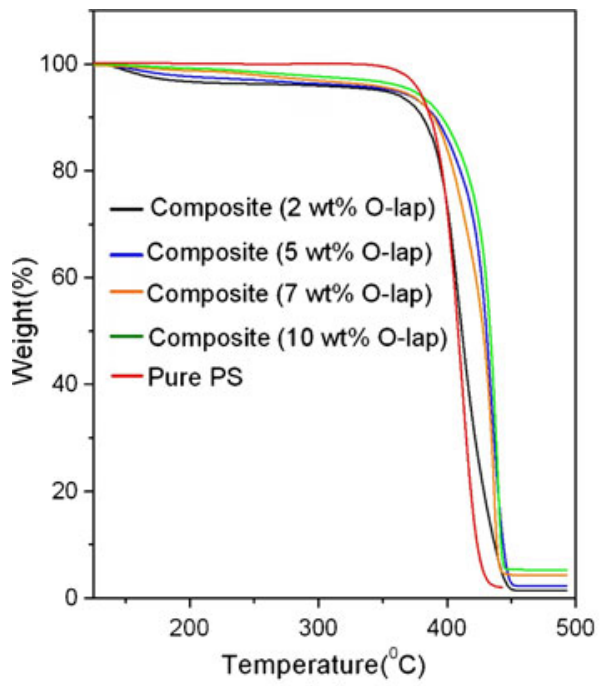

(a)

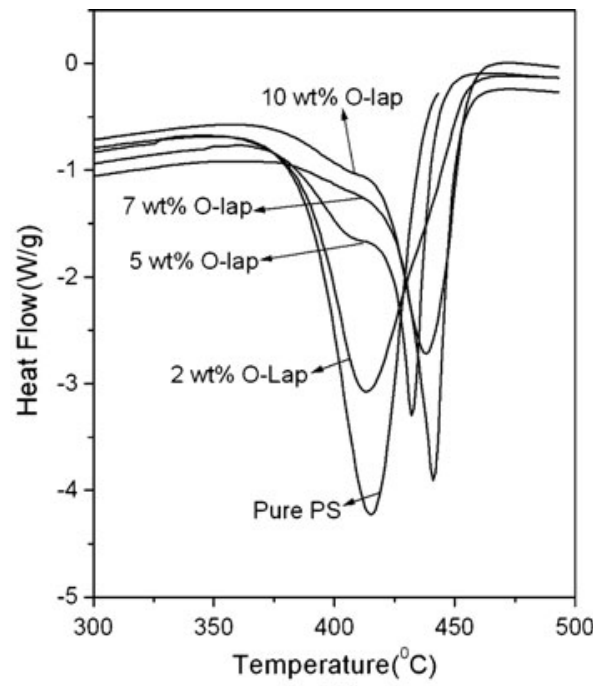

(b)

Figure 5. (a) TGA thermograms of pure PS along with composites having different amounts of O-lap. (b) DSC curves of pure PS along with composites having different amount of O-laponite.

shown in figure 5(a). In the figure, we observe that the thermal degradation temperature of pure PS is $425^{\circ} \mathrm{C}$, whereas it increases gradually (up to $454{ }^{\circ} \mathrm{C}$ ) in case of PS/O-laponite nanocomposites with increase in organo-laponite content. After this transition temperature in all the cases, there are no mass losses as revealed in this figure. The role of clay in the nanocomposite structure may be the main reason for the difference in TGA results of these systems compared to the previously reported systems (Ray and Okamoto 2003). Actually, polymer confinement and attraction to the clay intergallery surface modified with surfactant increases the necessary energy input to commence degradation. Clay acts 
as a heat barrier, which enhances the overall thermal stability of the system. Although clay is known to be concentrated on the exterior surfaces of PS particles, it seems that the presence of clay still plays an important role in enhancing the thermal stabilities of PS, by hindering the out-diffusion of the volatile decomposition products.

DSC results of both PS and PS/O-laponite nanocomposites are shown in figure 5(b). The glass transition temperature $\left(T_{\mathrm{g}}\right)$ in case of pure unmodified PS was found to be $379{ }^{\circ} \mathrm{C}$, whereas it gradually increases in case of PS/Olaponite nanocomposites with increase in the amount of Olaponite in the resultant composites (Noh and Lee 1999). Such an increase in $T_{\mathrm{g}}$ indicates that there was a strong interaction between PS matrix and laponite nanosheets and because of this, mobility of the PS chains near the interface was suppressed (Hou et al 2004). Since $T_{\mathrm{g}}$ is sensitive to the flexibility of the polymer chains and their local environment, increase in $T_{\mathrm{g}}$ could be attributed to reduced mobility of polymer chains in the vicinity of the clay platelets (Gilbert 1972). Additionally, a new phase transition is observed at $408{ }^{\circ} \mathrm{C}$ in case of PS/O-laponite composites having laponite content of $5 \mathrm{wt} \%$ and above. This may be due to the fact that at higher clay concentration, a large number of PS segments are anchored to the clay surface. As a result, this does not contribute to the melting of PS. This is also likely due to the conformation typical of amorphous PS (Lisi et al 2007).

\section{Conclusions}

In conclusion, our results reveal that laponite suitably modified by surfactant CTAB can be dispersed into PS matrix by solution intercalation technique to prepare stable PSlaponite nanocomposites. XRD results confirm that the characteristic peak (001) of pure laponite was shifted to lower angles which corresponds to an increase in $d$-spacing of about $9 \mathrm{~nm}$. In case of PS-laponite composites having 2 and $5 \mathrm{wt} \%$ O-laponite, there exists some mixture of intercalated and exfoliated structures of clay as evidenced from the XRD analysis. However, for higher laponite content, aggregation or agglomeration of clays occur in the composites and is manifested as the occurrence of new peak centred at around $2 \theta=11^{\circ}$. FTIR data reveals different types of vibrational signature of bonds between different species in the resultant composite materials. Presence of CTAB molecules in the interlayer spacings of laponite was confirmed by the occurrence of peaks at around $2923-2860 \mathrm{~cm}^{-1}$ and $1475 \mathrm{~cm}^{-1}$. FESEM image gives a clear visual evidence for the structural morphology of the prepared PS-laponite nanocomposites. The overall thermal stability of PS-laponite nanocomposites was increased as a function of O-laponite content in the mixture as is evidenced from the TGA results. DSC results also indicate the increase of glass transition temperature $\left(T_{\mathrm{g}}\right)$ of composites when compared to that of pure PS. Interestingly for higher clay concentration, a new phase transition is observed at around $408^{\circ} \mathrm{C}$ and is possibly due to the fact that a large number of PS segments are anchored to the clay surface at larger clay concentration. As a consequence, this cannot contribute to the melting of amorphous PS.

\section{Acknowledgements}

One of the authors (PKP) is grateful to UGC for providing financial support through UGC Minor Research Project (Sanction Ref. No. 39-983/2010(SR)) to perform this work and also to Jadavpur University for excellent infrastructural facilities. (SAH) also acknowledges DAE for financial support through DAE Young Scientist Research Award (No. 2009/20/37/8/BRNS/3328).

\section{References}

Alexandre M and Dubois P 2000 Mater. Sci. Eng. 281

Aranda P and Ruiz-Hitzky E 1992 Chem. Mater. 41395

Biswas M and Ray S S 2001 Adv. Polym. Sci. 155167

Blanton T N, Majumdar D and Melpolder S M 1998 Adv. X-ray Anal. 42562

Bourbigot S, Le B M, Dabrowski F, Gilman J W and Kashiwagi T 2000 Fire Mater. 24201

Daniel L M, Ray L F and Huai Y Z 2008 J. Colloid Interf. Sci. 321302

Doeff M M and Reed J S 1998 Solid State Ionics 109113

Eduardo R H and Pilar A 1990 Adv. Mater. 2545

Fang F F, Kim J H, Choi H J and Kim C A 2009 Colloid Polym. Sci. 287745

Fossum J O 1990 Physica A 270270

Frost R L, Daniel L M and Zhu H 2007 J. Colloid Interf. Sci. 31672

Fu X A and Qutubuddin S 2004 Polym. Eng. Sci. 44345

Giannelis E P 1996 Adv. Mater. 829

Gilbert P 1972 Proc. R. Soc. Ser. B 18289

Greenland D J 1963 J. Colloid Sci. 18647

Guimarães A M F, Ciminelli V S T and Vasconcelos W L 2007 Mater. Res. 1037

Hanley H J M, Muzny C D and Butler B D 1997 Langmuir 135276

Hou W, Zhao W and Li D 2004 Chin. J. Polym. Sci. 22459

Lisi R D, Lazzara G, Milioto S and Muratore N 2007 J. Therm. Anal. Calorim. 8761

Noh M W and Lee D C 1999 Polym. Bull. 42619

Ravichandran J, Manoj A G, Liu J, Manna I and Carroll D L 2008 Nanotechnology 19085712

Ray S S 2006 J. Ind. Eng. Chem. 12811

Ray S S and Okamoto M 2003 Prog. Polym. Sci. 281539

Ray S, Quek S Y, Easteal A and Chen X D 2006 Int. J. Food. Eng. 21

Ruggerone R, Plummer C J G, Negrete H N, Bourgeat-Lamib E and Månson J A E 2009 Eng. Fract. Mech. 762846

Sun Q, Deng Y and Wang Z L 2004 Macromol. Mater. Eng. 289288

Szabó T, Mitea R, Leeman H, Premachandra G S, Johnston C T, Szekeres M, Dekany I and Schoonheydt R A 2008 Clays Clay Miner. 56494

Tawari S L, Koch D L and Cohen C 2001 J. Colloid Interf. Sci. 240 54

Velde B 1992 Introduction to clay minerals (London: Chapmann and Hall)

Wang X, Pang S, Yang J and Yang F 2006 Trans. Nonferr. Met. Soc. China 16524 